\title{
Low-frequency Slivan states in the outer main belt?
}

\author{
J. Vraštil and D. Vokrouhlický \\ Institute of Astronomy, Charles University, V Holešovičkách 2, 18000 Prague 8, Czech Republic \\ e-mail: vrastil@sirrah.troja.mff.cuni.cz; vokrouhl@cesnet.cz
}

Received 14 October 2015 / Accepted 5 December 2015

\begin{abstract}
Context. Spin states of several main belt asteroids have been recently found to reside in what is called the Slivan state, namely a secular spin-orbit resonance with the $s_{6}$ mode of their orbital precession in space.

Aims. We examine a possibility of the Slivan states of asteroids with other than orbital $s_{6}$ precession frequency.

Methods. The asteroids' orbital and spin states are numerically propagated using well-tested computer codes. We select parameter space favorable for the Slivan-state capture with the $s_{7}$ frequency mode of the orbital precession. The stability of these states is numerically verified.

Results. We find that asteroid (184) Dejopeja has a spin state captured in (or very nearly) the Slivan state with the $s_{7}$ orbital frequency. In general, such a situation may favorably occur for low-inclination orbits in the outermost part of the main asteroid belt. We expect these states to be common among the Themis family members.
\end{abstract}

Key words. celestial mechanics - minor planets, asteroids: general

\section{Introduction}

Large amounts of accurate astronomical observations as well as rapid advancements in the methods of their analysis have recently lead to a significant leap in knowledge of the rotational motion of small bodies in the solar system (e.g., Durech et al. 2015, and references therein). Here we are mainly interested in the main belt asteroids. The Asteroid Lightcurve Data Base ${ }^{1}$ (LCDB) contains recent information for more than 15000 objects, of which nearly 4000 have a very accurately determined rotation period. Additionally, the spin state and shape models are available for nearly 400 asteroids (e.g., Durech et al. 2010), and all these numbers are rapidly increasing every year. This high-quality dataset resulted in analyses that reveal interesting processes that make the asteroid spin states evolve on various timescales.

One branch of these studies originated from the works of Skoglöv and his collaborators about two decades ago (e.g., Skoglöv et al. 1996; Skoglöv 1997, 1998). While planting a seed of interest, these works were mainly of a theoretical nature without immediate applications in mind. The situation changed after a detailed characterization of spin states for about a dozen large asteroids in the Koronis family (Slivan 2002; Slivan et al. 2009). These results were very unusual; the most surprising finding was that all prograde-rotating bodies have nearly parallel spin axes in the inertial space and also have similar rotation periods. Soon, Vokrouhlický et al. (2003) came up with a model able to explain the situation. They proposed that the prograde-rotating Koronis asteroids from the Slivan sample are captured in a secular spin-orbit resonance and they called the configuration "Slivan states" (see Sect. 2). In this case, the resonance implied 1:1 commensurability between the general

\footnotetext{
1 http: //www . minorplanet . info/lightcurvedatabase. html and Warner et al. (2009).
}

precession of an asteroid's spin axis due to the solar torque and the $s_{6}$ frequency mode with which the orbital plane precesses in space. Additionally, Vokrouhlický et al. (2003) showed that this situation is a natural end-state of a billion-year evolution driven by the Yarkovsky-O'Keefe-Radzievskii-Paddack (YORP) effect. Recently, Kryszczyńska (2013) suggested that similar Slivan states might also exist among the Flora-family members at a much smaller heliocentric distance. However, Vraštil \& Vokrouhlický (2015) showed that their stability is much lower than in the Koronis-family zone, and proposed instead that asteroids in the Massalia family are better situated to have their axes captured in the stable Slivan states. A general overview of conditions favorable for Slivan states in the main belt, as well other aspects of the asteroid long-term spin dynamics, were also studied by Vokrouhlický et al. (2006). Finally, returning to the roots set by the Skoglöv group, Vokrouhlický et al. (2005) showed that the second largest near-Earth asteroid, (433) Eros, has its spin axis captured in the secular spin-axis resonance similar to the Koronis bodies but now with the proper frequency mode $s$ of the orbital precession. The stability of this configuration is even lower than in the Flora-asteroids case, so Vokrouhlický et al. (2005) were not able to draw conclusions about the past evolutionary trajectory of Eros' spin axis and the processes that were in action.

Overall, the conditions for the stable Slivan states were found to be most favorable among the low-inclination orbits in the outer parts of the asteroid belt, which is caused because obliquities of asteroids residing on the high-inclination orbits necessarily oscillate with large amplitude (comparable to the orbital proper inclination). With this geometric effect, confinement in the Slivan states is broken (e.g., Vokrouhlický et al. 2003; Vraštil \& Vokrouhlický 2015). So far, however, the studied Slivan states have implied resonance with the prominent (largest-amplitude) mode $s_{6}$ of the orbital plane precession in space. Moving to larger heliocentric distances could mean that lower frequencies participating in orbital plane precession become more important. 
Indeed, the giant planets spin axes are primarily influenced by the $s_{7}$ frequency (in the case of Jupiter; Ward \& Canup 2006; Vokrouhlický \& Nesvorný 2015) or even the $s_{8}$ frequency (in the case of Saturn; Ward \& Hamilton 2004; Hamilton \& Ward 2004; Boué et al. 2009; Vokrouhlický \& Nesvorný 2015). Henceforth, in this paper we analyze the role of the $s_{7}$ frequency term in the orbital precession of the outer main belt asteroids for their longterm spin dynamics. We show that the orbital zone occupied by the large Themis family is a particularly interesting region in which to search for possible Slivan states associated with this lower frequency. As an example, we suggest that the spin state of (184) Dejopeja may be close to, or reside in, such a resonance.

The paper is organized as follows. In Sect. 2 we briefly summarize the theory needed to understand the secular spin-axis evolution of asteroids; an interested reader may find more details in the previous literature (e.g., Vokrouhlický et al. 2006; Vraštil \& Vokrouhlický 2015). We also describe numerical tools and codes used in our study. In Sect. 3 we motivate the case for possible low-frequency Slivan states using the outer main belt asteroid (184) Dejopeja, and in Sect. 4 we show how these states could result from the YORP driven evolution of small members in the Themis family.

\section{Methods}

We assume an asteroid rotating about the shortest axis of its inertia tensor. Denoting the direction of the spin axis by a unit vector $s$, one easily verifies that its long-term evolution satisfies (e.g., Bertotti et al. 2003)

$$
\frac{\mathrm{d} s}{\mathrm{~d} t}=-\alpha(\boldsymbol{c} \cdot \boldsymbol{s})(\boldsymbol{c} \times \boldsymbol{s}) .
$$

This model considers gravitational torque by the Sun exerted on the asteroid rotational motion, where $c$ is the unit vector in the direction of the orbital angular moment and

$\alpha=\frac{3}{2 \eta^{3}} \frac{n^{2}}{\omega} \Delta$,

where $\eta=\sqrt{1-e^{2}} ; e$ is the orbital eccentricity; $n$ is the orbital mean motion; and $\Delta$ is the dynamical ellipticity of the body, which depends on the principal values $(A, B, C)$ of the inertia tensor

$\Delta=\frac{C-\frac{1}{2}(A+B)}{C}$

(we assume $A \leq B \leq C$ ). As an example, the low-eccentricity orbits with $e \ll 1$ in the outer part of the asteroid belt with semimajor axis $a \simeq 3.1$ au have numerically (e.g., Vokrouhlický et al. 2006)

$\alpha \simeq 44.6 \Delta P_{6}^{\prime \prime} / \mathrm{yr}$,

where $P_{6}=P / 6 \mathrm{~h}$ is the rotation period $P$ expressed nondimensionally in units of $6 \mathrm{hr}$ (characteristic of many asteroids).

Equation (1) assumes all vectors given in an inertial frame. While the precession parameter $\alpha$ is approximately constant, $c$ is not. This occurs because the orbital plane evolves as a result of planetary perturbations. As it is a unit vector, $c$ can be expressed using two scalar parameters such as the orbital inclination $I$ and longitude of node $\Omega$ or, in a non-singular way, using the components of a complex variable $\zeta=\sin I / 2 \exp (l \Omega)$. All these parameters are generally time dependent.
If $s$ and $c$ are expressed with respect to the osculating orbital frame in which $\boldsymbol{c}^{T}=(0,0,1)$, when the reference $x y$ plane coincides with orbital plane, Eq. (1) takes the following form:

$\frac{\mathrm{d} \boldsymbol{s}}{\mathrm{d} t}=-[\alpha(\boldsymbol{c} \cdot \boldsymbol{s}) \boldsymbol{c}+\boldsymbol{h}] \times \boldsymbol{s}$.

The simplification in $c$ is compensated with the occurrence of a new vectorial parameter $\boldsymbol{h}^{T}=(\mathcal{A}, \mathcal{B},-2 C)$, with

$$
\begin{aligned}
\mathcal{A} & =\cos \Omega \dot{I}-\sin I \sin \Omega \dot{\Omega}, \\
\mathcal{B} & =\sin \Omega \dot{I}+\sin I \cos \Omega \dot{\Omega}, \\
C & =\sin ^{2} I / 2 \dot{\Omega},
\end{aligned}
$$

where the overdots mean time derivatives. The newly appearing torque $-\boldsymbol{h} \times \boldsymbol{s}$ expresses non-inertial effects in the moving reference frame. Returning to the unit vector $s$, we note that it can also be expressed using two scalar parameters. Usually one chooses

$$
s=\left(\begin{array}{c}
\sin \varepsilon \sin \psi \\
\sin \varepsilon \cos \psi \\
\cos \varepsilon
\end{array}\right)
$$

where $\varepsilon$ is the obliquity and $\psi$ the precession angle.

Solving Eq. (5) or equivalent equations for $\varepsilon$ and $\psi$ may still be very complicated because the orbit evolution is complicated. Typically, $\zeta(t)$ consists of many Fourier terms, thus $\zeta(t)=\sum A_{k} \exp \left(\imath \Omega_{k}\right)$, each of which has a constant amplitude $A_{k}$ (i.e., associated inclination value $A_{k}=\sin I_{k} / 2$ ) and frequency $\dot{\Omega}_{k}=s_{k}$. Because the perturbing planets themselves interact with each other, some of the $s_{k}$-frequencies are transferred to the asteroid orbital plane precession from their motion. The characteristic spectrum of $s_{k}$ frequencies for an asteroid thus consists of (i) a proper mode, associated with free initial conditions of the orbital motion and denoted by $s$; and (ii) the planetary terms. The latter are dominated by the effects of giant planets and they are typically denoted by $s_{6}, s_{7}$, and $s_{8}$. Their numerical values are $s_{6} \simeq-26.34^{\prime \prime} / \mathrm{yr}, s_{7} \simeq-2.99^{\prime \prime} / \mathrm{yr}$, and $s_{8} \simeq-0.69^{\prime \prime} / \mathrm{yr}$ in decreasing frequency (e.g., Laskar 1988). The largest amplitude (inclination) is associated with the fastest frequency $s_{6}$ because this is principally due to mutual interaction of the gas giants. More distant ice giants, Uranus and Neptune, contribute with slower frequencies $s_{7}$ and $s_{8}$. There are also many Fourier terms in $\zeta$ that correspond to linear combinations of the principal components described above. Their amplitudes are, however, generally very small.

In the first approximation, one can imagine the solution of Eq. (5) as a linear combination of partial solutions for each of the individual terms in the Fourier decomposition of $\zeta$ (especially valid when the frequencies $s_{k}$ are not close each other). These partial solutions, where simply $\zeta=\sin I / 2 \exp [l(s t+\phi)]$, are integrable and are known as a Colombo top problem (e.g., Colombo 1966; Henrard \& Murigande 1987). The most interesting feature of the Colombo top is the occurrence of stationary solutions. Their number depends on a non-dimensional parameter $\kappa=\alpha /(2 s)$. In a simpler situation, when $|\kappa|<\kappa_{\star}$, there are two stationary solutions, otherwise there are four stationary solutions (traditionally known as Cassini states). The threshold value for $\kappa$ reads (see, e.g., Henrard \& Murigande 1987)

$\kappa_{\star}=\frac{1}{2}\left(\sin ^{2 / 3} I+\cos ^{2 / 3} I\right)^{3 / 2}$.

For low-inclination cases $\kappa_{\star} \simeq \frac{1}{2}$, and the two new stationary solutions bifurcate when $\alpha \simeq-s$. While stationary with respect to 
the moving frame with nodal longitude $\Omega=s t+\phi$, the Cassini states obviously regularly precess in the inertial space. Their obliquity value is given by solutions of the transcendental equation (see, e.g., Colombo 1966; Henrard \& Murigande 1987)

$\kappa \sin 2 \varepsilon=-\sin (\varepsilon \mp I)$

with the upper sign - for $\varphi=0^{\circ}$ and lower sign + for $\varphi=180^{\circ}$; the definition of the longitude in the moving frame is $\varphi=-(\psi+$ $\Omega$ ) and it reckons from a direction $90^{\circ}$ from the ascending node. Of particular interest is $\varphi=0^{\circ}$ stationary point when $|\kappa|>\kappa_{\star}$, which is usually referred to as Cassini state $2\left(\mathrm{C}_{2}\right)$, because it has the character of a stable resonant state: small perturbations make obliquity oscillate about $\varepsilon_{2}$ and longitude $\varphi$ librate about zero. The nature of the resonance is seen from Eq. (9) whose limit for $I \simeq 0$ becomes $\kappa \sin 2 \varepsilon \simeq-\sin \varepsilon$. The obvious solutions $\varepsilon_{1} \simeq 0^{\circ}$ and $\varepsilon_{3} \simeq 180^{\circ}$ correspond to Cassini states 1 and 3 , while Cassini states 2 and 4 are at approximately $\alpha \cos \varepsilon_{2,4} \simeq-s$. The left-hand side is the regular precession of $s$ produced by the gravitational torque of the center, while the right-hand side is the orbital precession rate. Thus the Cassini state 2 resonance expresses 1:1 commensurability between the two. The maximum width $\Delta \varepsilon$ of the resonant zone associated with Cassini state 2 can be determined from (e.g., Henrard \& Murigande 1987; Ward \& Hamilton 2004; Vokrouhlický et al. 2006)

$\sin \frac{\Delta \varepsilon}{2}=\frac{1}{|\kappa|} \sqrt{\frac{\sin 2 I}{\sin 2 \varepsilon_{4}}}$,

where $\varepsilon_{4}$ is obliquity of the unstable equilibrium from Eq. (9). An important implication of the square-root factor on the righthand side of Eq. (10) is that $\Delta \varepsilon$ may be significant (e.g., degrees) even for very small values of $I$ (e.g., a small fraction of a degree).

We can now combine the precession constant $\alpha$ from Eq. (4) with the Cassini state 2 location from $\alpha \cos \varepsilon_{2} \simeq-s_{k}$ to probe its onset and location at the outer part of the main belt ( $a \simeq 3.1$ au and $e, I \ll 1$ ) for different frequencies $s_{k}$ expected in the orbital evolution. We ignore the proper frequency $s$, which is too high in this orbital zone. Indeed, $s \sim-100^{\prime \prime} / \mathrm{yr}$ such that even for the extreme $\Delta \simeq \frac{1}{2}$ value the Cassini resonance associated with $s$ would require long rotation periods $P \geq 28 \mathrm{~h}$. Instead, we focus on the case of planetary frequencies $s_{6}$ and $s_{7}$. Figure 1 shows correlated values of $\Delta$ and $P$ for which the resonant Cassini state 2 associated with both frequencies onsets, and in the case of $s_{7}$ frequency its location at $\varepsilon_{2}=30^{\circ}, 50^{\circ}$, and $70^{\circ}$. Asteroids with sizes between one kilometer and tens of kilometers have a characteristic $\Delta$ value between 0.1 and 0.3 (e.g., Vokrouhlický \& Čapek 2002). Bodies with a sufficiently short rotation period, between a few hours and about a day, would also avoid their rotation states to be captured in the traditional Slivan state associated with the precession frequency $s_{6}$. However, a theoretical possibility occurs for bodies with short enough rotation period to have their spin axis located in a Cassini state 2 resonance zone for the lower frequency $s_{7}$. As an example, we show the location of asteroid (184) Dejopeja in this parametric space (see also Sect. 3).

In the next section we numerically integrate Eq. (5) to investigate this suggested possibility of low-frequency Slivan states for outer main belt asteroids and to analyze their stability. We make use of an efficient symplectic integrator proposed by Breiter et al. (2005), in particular adopting the LP2 splitting scheme. Since we deal with the secular evolution of $s$ we choose a conveniently long timestep of $50 \mathrm{yr}$. The code needs time series describing the orbital evolution of the asteroid, namely the values of the semimajor axis $a$, eccentricity $e$, and the complex

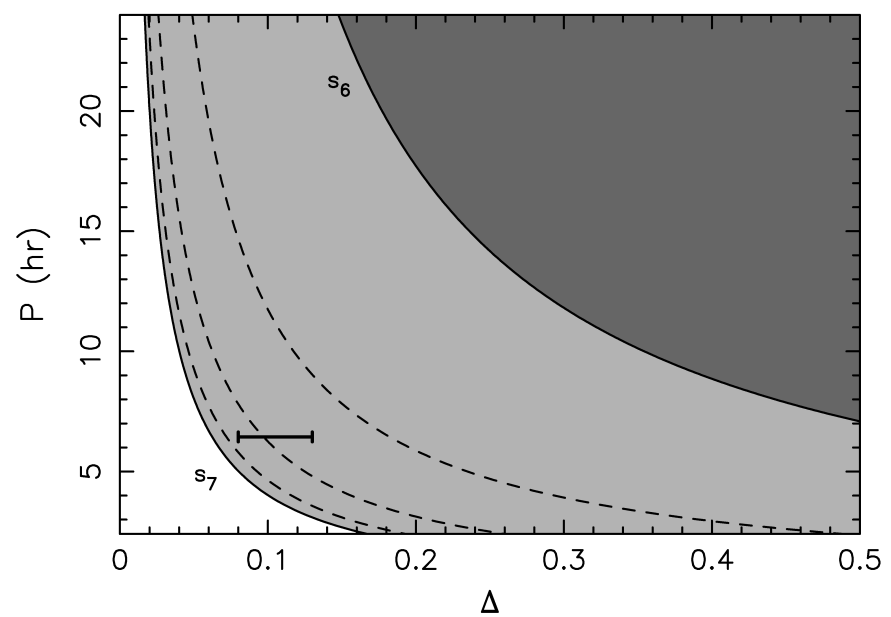

Fig. 1. Occurrence of spin-orbit resonances (Slivan states) for lowinclination and low-eccentricity asteroid orbits with semimajor axis of 3.1 au: dynamical ellipticity $\Delta$ at the abscissa vs. rotation period $P$ at the ordinate (in hours). The solid lines labeled $s_{6}$ and $s_{7}$ denote location where the Slivan state with the appropriate nodal frequency exists at near-to-zero obliquity in this parameter space. Both states exist in the dark gray zone, while only the $s_{7}$ state exists in the light gray zone (even lower $s_{k}$-frequencies would be needed in the white zone). The dashed lines show where the resonance Cassini state 2 of the $s_{7}$ frequency has obliquity $30^{\circ}, 50^{\circ}$, and $70^{\circ}$, from left to right. The horizontal line segment is the location of (184) Dejopeja; the uncertainty in $P$ is too small to be visible, so basically the estimated uncertainty in $\Delta$ is shown.

parameter $\zeta$ that combines the longitude of the node and the inclination. This information is obtained using the well-tested and widely used integration package ${ }^{2}$ swift. Because swift integrates a full system of equations of motion for both planets and asteroid(s), it requires a shorter timestep. We typically used 5 days, short enough to realistically describe the orbital evolution of all bodies (including the planet Mercury). Initial orbital state vectors for the chosen asteroids and a given epoch were taken from the AstDyS internet database ${ }^{3}$, and for the planets from the JPL DE405 ephemerides file. To organize the propagation efficiently, we embedded our secular spin integration scheme into the swift package. This arrangement not only allows the spin evolution to be propagated online, which avoids large output files with the orbital evolution, but it also allows the spin evolution of several asteroids or parametric variants of the same asteroid (for instance testing evolution for different values of the dynamical ellipticity parameter $\Delta$ ) to be propagated simultaneously. We note that the spin propagation only needs at a given time to know the orbital parameters in the neighboring grid-points in time, readily provided by the swift integrator.

\section{3. (184) Dejopeja}

Asteroid (184) Dejopeja belongs to a large group of objects discovered by Johann Palisa between 1870s and $1920 \mathrm{~s}^{4}$

\footnotetext{
2 http://www.boulder.swri.edu/ hal/swift.html

3 http://hamilton.dm.unipi.it/astdys/

4 Interestingly, the original name, Deiopeia, given by Palisa in his 1878 discovery paper changed to Dejopeja in Palisa (1879), and remained this way ever since then (the change may have to do with Palisa's appointment as director of the Naval Observatory in Pula, today's Croatia). In fact, the original Greek spelling would have been Deiopea, one of the goddess Juno's nymphs famously known from Virgil's Aeneid: ... Succeed my wish, and second my design; The fairest, Deiopea, shall be thine, ...
} 
(Palisa 1878). As in the case of many similar asteroids, after an uneventful century of merely collecting astrometric data, planetary astronomy started to provide physical data about Dejopeja at the end of the last century. Spectral observations allowed it to be classified as an X-type object (e.g., Bus \& Binzel 2002). Measurements of several space infrared missions provided size estimates between $D \simeq 66 \mathrm{~km}$ (IRAS and AKARI; Tedesco et al. 2002; Usui et al. 2011) and $D \simeq 88 \mathrm{~km}$ (WISE; Masiero et al. 2011), and correspondingly large or intermediate values of geometric albedo. The rapid advancement in asteroid population knowledge (computational methods allowing precise proper orbital elements to be determined and clustering techniques aided by color information) allowed Dejopeja to be classified as a background object without an evident link to any of the known asteroid families. The closest significant family is Themis, whose members have on average slightly larger proper eccentricity and slightly smaller proper inclination than Dejopeja (e.g., Nesvorný et al. 2015); however, they share the same heliocentric distance.

Finally, and the most relevant for our work, photometric observations and advanced lightcurve inversion techniques allowed Dejopeja's rotation state to be determined and its shape to be roughly constrained. We adopt here the spin-state solution from Marciniak et al. (2007), which has a sidereal rotation period of $6.44111 \mathrm{~h}$ and two possible pole orientations, namely $(\lambda, \beta)=\left(200^{\circ}, 52^{\circ}\right)($ pole $\mathrm{P} 1)$ and $(\lambda, \beta)=\left(18^{\circ}, 54^{\circ}\right)($ pole $\mathrm{P} 2)$ with $\lambda$ and $\beta$ ecliptic longitude and latitude. The associated shape models for P1 and P2 are roundish with formal values of $\Delta$ equal to 0.11 (assuming uniform density distribution). However, the dynamical ellipticity $\Delta$ is the least accurately determined parameter from the lightcurve inversion procedure. A typical uncertainty in $\Delta$ may be between $30-50 \%$, so a realistic interval of values of this parameter for Dejopeja may be 0.07-0.15.

As a pre-requisite of our work we numerically integrated the nominal heliocentric orbit of Dejopeja for a $10 \mathrm{Myr}$ interval of time. The initial conditions were taken from the AstDyS site. We used the swift code, an integration timestep of 5 days, and output every 50 years. Next, we used the algorithm described in Ferraz-Mello (1981) to determine the frequency spectrum of the non-singular inclination element $\zeta=\sin I / 2 \exp (\imath \Omega)$. Figure 2 shows the result. Typical of most asteroids, the principal spectral contribution is from the proper term with frequency $s \simeq-96.2^{\prime \prime} / \mathrm{yr}$ and amplitude $I \simeq 2.1^{\circ}$ (see also AstDys site). The next three contributing lines are the expected planetary terms $s_{6}, s_{8}$, and $s_{7}$, listed here in order of decreasing amplitudes. The largest of them is $I_{6} \simeq 0.45^{\circ}$, but we are mainly interested in the $s_{7} \simeq-2.985^{\prime \prime} / \mathrm{yr}$ line with an amplitude of $I_{7} \simeq 0.06^{\circ}$ and phase $\phi_{7} \simeq 145^{\circ}$ at the initial epoch J2000.0 of our integrations. We note that this sets the $\varphi=0^{\circ}$ meridian of the associated Cassini state 2 at the ecliptic longitude of about $235^{\circ}$, not far from Dejopeja's pole P1 value. Assuming P1 is the correct pole position for Dejopeja, it would have $\varphi \simeq-35^{\circ}$ in the $s_{7}$-frequency precessing frame. Given negligible inclination $I_{7}$, the obliquity in the frame would be essentially equal to the osculating value $\varepsilon \simeq 38^{\circ}$. Obviously, if $\mathrm{P} 2$ is the correct pole position, we would have $\varphi \simeq-127^{\circ}$ and $\varepsilon \simeq 36^{\circ}$, less favorably located with respect to Cassini state 2 of the $s_{7}$-frequency frame.

We can now use Fig. 1 and Eq. (10) to estimate the odds that Dejopeja's pole P1 resides in the Slivan state associated with the $s_{7}$ frequency. Given $\varepsilon_{4} \simeq 40^{\circ}, I_{7} \simeq 0.06^{\circ}$, and assuming the best case of $|\kappa| \simeq 0.5$, we obtain the maximum resonance width $\Delta \varepsilon \simeq 10^{\circ}$. Because $\mathrm{P} 1$ has a small value of $\varphi$, basically the whole interval $\Delta \varepsilon$ is available to situate P1 into the resonance zone. Obviously, $\varepsilon_{2}$ depends on $\Delta$. Figure 1 indicates that

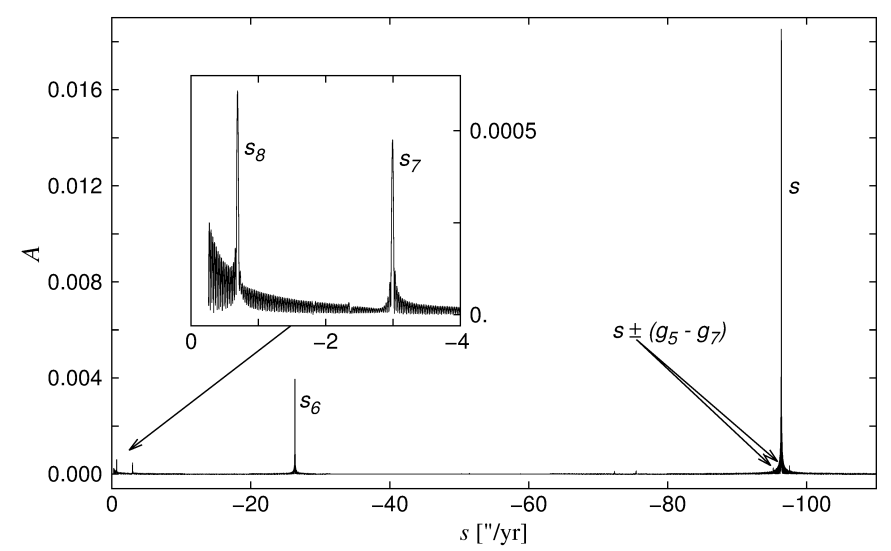

Fig. 2. Frequency spectrum of $\zeta=\sin I / 2 \exp (\imath \Omega)$ from a $10 \mathrm{Myr}$ numerical orbital integration of (184) Dejopeja. The abscissa is the frequency in arcseconds per year, and the ordinate in amplitude of the quasiperiodic approximation $A \exp [\imath(s t+\phi)]$. The principal spectral lines are labeled using the standard notation in planetary studies: (i) $s$ is the proper frequency; (ii) $s_{6}, s_{7}$, and $s_{8}$ are the linear planetary frequencies.

$\varepsilon_{2} \simeq 35^{\circ}-40^{\circ}$ when $\Delta \leq 0.09$, but becomes larger for $\Delta>0.09$. As a result, if Dejopeja's $\Delta$ value is at the lower end of our estimated interval $0.07-0.15$, the pole position would qualify it to reside in the Cassini resonance zone.

Armed with the analytical estimates, we now use our numerical code that combines swift and Breiter et al. (2005) integrators to propagate Dejopeja's spin axis starting from the P1 pole position at the current epoch. We used a 5-day integration timestep for the orbital component and 50-year timestep for the spin component. The integration timespan was $50 \mathrm{Myr}$. We tested a number of $\Delta$ values in the expected interval $0.07-0.15$ and analyzed how the results depend on its choice. Figure 3 shows a sample of these results: Dejopeja's spin axis evolution, initially equal to the $\mathrm{P} 1$ pole, has been projected onto the reference frame defined by the node $\Omega_{7}$ and inclination $I_{7}$ of the corresponding term in $\zeta$. As expected, for low enough values of $\Delta$ the pole is safely captured in the resonance zone of Cassini state 2 and librates about $\mathrm{C}_{2}$. The characteristic libration period is $\sim 20 \mathrm{Myr}$ and perturbations from other Fourier terms in $\zeta$, principally $s$ and $s_{6}$, are small enough not to break the resonance lock. We estimate that the pole resides in the resonant zone for $\Delta$ values between 0.081 and 0.092 . As expected, when $\Delta \geq 0.092$ the forced obliquity of Cassini state 2 migrates to larger values, leaving the pole P1 outside the associated libration region. This results in simple circulation of P1 about Cassini state 1 (rightmost panel in Fig. 3). Figure 4 shows the time evolution of the osculating obliquity $\varepsilon$ of Dejopeja for the four values of $\Delta$ shown in Fig. 3. The middle panels, in particular, show large oscillations in $\varepsilon$ triggered by the resonant phenomenon and have a $\sim 20 \mathrm{Myr}$ period of librations around $\mathrm{C}_{2}$.

We also verified that similar numerical integrations starting initially from the pole $\mathrm{P} 2$ generally avoid being captured in the resonant zone around $\mathrm{C}_{2}$ of the $s_{7}$ frequency. Only when $\Delta$ is in a very narrow interval of values between 0.080 and 0.081 does it have a very large amplitude libration regime around $\mathrm{C}_{2}$ because its initial longitude $\varphi \simeq-127^{\circ}$ with respect to $C_{2}$ is large. 

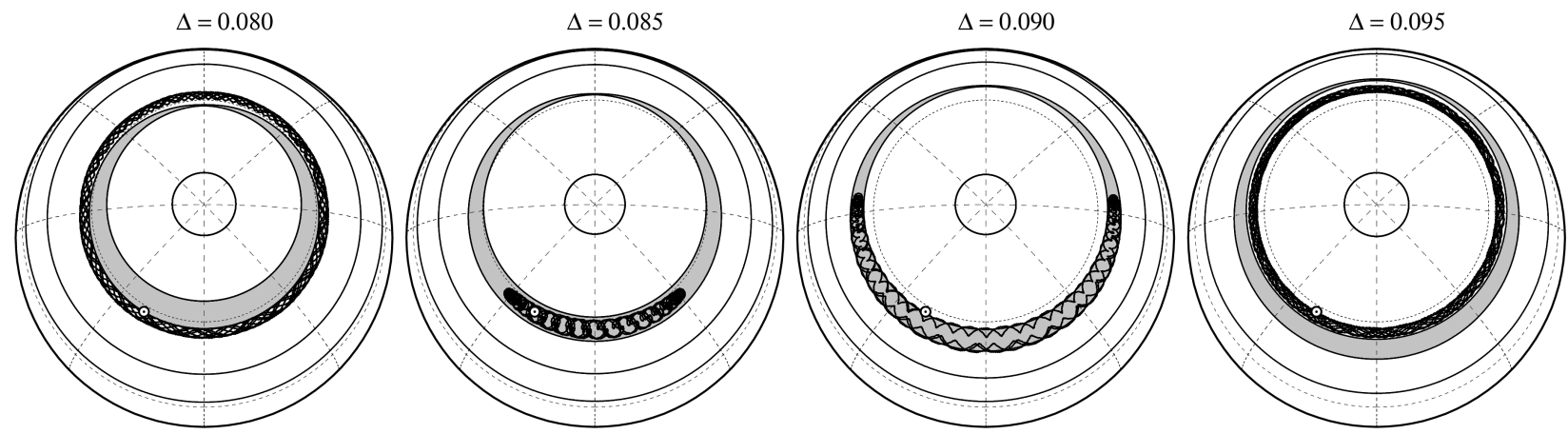

Fig. 3. Evolution of the rotation pole P1 for (184) Dejopeja (symbols) projected onto the reference frame defined by nodal longitude $\Omega_{7}$ and inclination $I_{7}$ of the $s_{7}$ frequency line in Fourier representation of $\zeta=\sin I / 2 \exp (\imath \Omega)$. The initial spin position is shown by the $\odot$ symbol. The projection is given on a celestial sphere viewed from an almost pole-on direction. The origin of longitude $\varphi, 90^{\circ}$ ahead of $\Omega_{7}$, is located downwards. Four panels are shown for four values of the dynamical ellipticity parameter $\Delta$ given at the top. The dashed lines show the meridian and latitude circles. If $\zeta$ were to contain only the $s_{7}$ frequency term (Colombo model), the spin evolution would follow the solid lines. In the middle panels the spin remains well confined in the resonant zone about the Cassini state $C_{2}$ highlighted in gray.

\section{Themis family orbital zone}

In the previous section, we verified that under the right parameter combination the outer belt asteroids could have their spin axes captured in the Slivan state associated with the low $s_{7}$ frequency. Equation (4) indicates that $\Delta P_{6} \simeq 0.07-0.2$, which requires small, but conceivable values of both ellipticity and the rotation period. Obviously, the preferred orbits should have low inclination because the high-frequency obliquity oscillations might break confinement in the Slivan state for large inclinations. However, the resonance zone of the low-frequency Slivan state is generally small (see Vraštil \& Vokrouhlický 2015, for computation details) and expecting that a spin axis of a given asteroid would occupy it at random is a very small probability event. If, however, long-term evolution of the spin state leads to a stable capture in the $s_{7}$ frequency related Slivan state, as in the case of the classical Slivan states of asteroids in the Koronis family (Vokrouhlický et al. 2003), we could expect more asteroids to reside in this low-frequency Slivan state. Interestingly, the favored orbital zone (outer main belt and low-inclination orbits) is occupied by a large Themis asteroid family. This may provide enough asteroids to test our hypothesis. Unfortunately, to date no reliable spin states are known for Themis members with size $<40 \mathrm{~km}$ because Themis asteroids are generally faint and observational efforts using small telescopes have not provided enough data yet. However, the situation may rapidly change in the near future when data from more powerful observational systems is available for spin-state reconstruction of the main belt asteroids.

For these reasons we now test the possibility of a stable capture in the low-frequency Slivan state using a hypothetical member in the Themis family. We actually use the orbit of (24) Themis itself, which is a good representative for all Themis members, but assume the strength of the YORP effect that would correspond to a smaller body (say $D<40 \mathrm{~km}$ ). Our numerical experiment is similar to that in Sect. 3.4 of Vraštil \& Vokrouhlický (2015). For the sake of simplicity we assume that the rotation period $P$ of the body slowly and steadily increases. This is one of the main secular effects of the YORP effect (e.g. Bottke et al. 2006). A Themis member $30 \mathrm{~km}$ in size that has an initially fast rotation of $\sim 3 \mathrm{~h}$ and low obliquity can typically have $\mathrm{d} P / \mathrm{d} t \sim(1-3) \times 10^{-3} \mathrm{~h} / \mathrm{Myr}$. This means it could increase its rotation period by $\sim 2-4 \mathrm{~h}$ in a few billion years, a likely age of this large family. In our simulation we initially set a rotation period of $3 \mathrm{~h}$ and zero obliquity, and we note that the results do

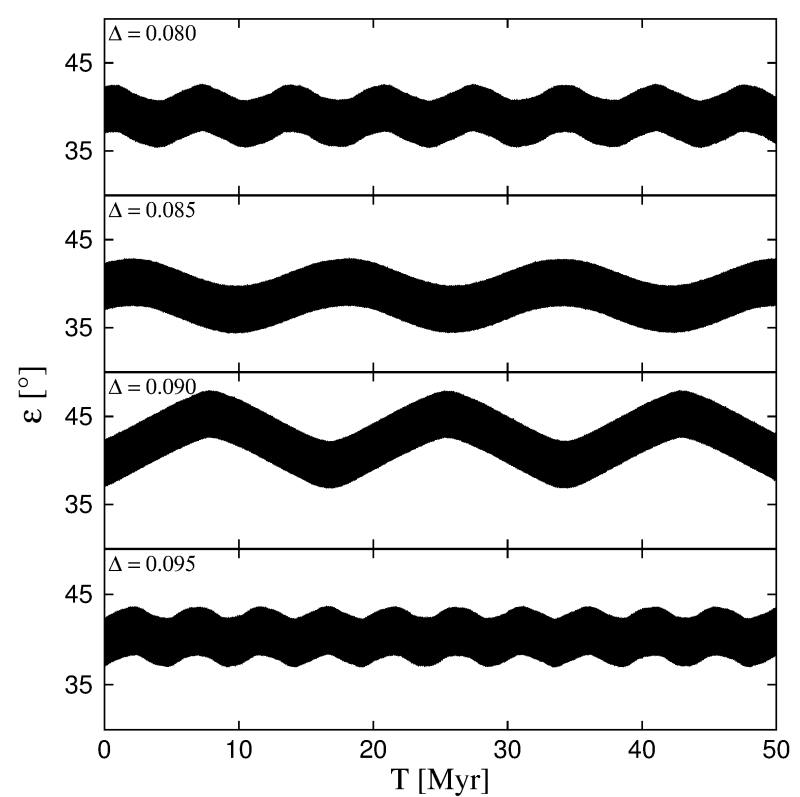

Fig. 4. Time evolution of the osculating obliquity $\varepsilon$ of Dejopeja for the four cases considered in Fig. 3. The resonant cases, middle panels, are characterized by large amplitude and long-period obliquity variations. These reflect librations in the Cassini resonance zone having $\Delta \varepsilon \simeq 10^{\circ}$ width.

not critically depend of the latter assumption (assuming $5^{\circ}-10^{\circ}$ obliquity would give basically the same results). We let the rotation period increase at a steady rate $\mathrm{d} P / \mathrm{d} t=2 \times 10^{-3} \mathrm{~h} / \mathrm{Myr}$ and use our code to simultaneously integrate the orbit and spin of the body for $1 \mathrm{Gyr}$.

Figure 5 shows selected time intervals of the spin evolution as provided by our simulation. To make the capture in the lowfrequency Slivan state easier to understand, we use the same coordinate frame as in Fig. 3. This means the origin of longitude is $90^{\circ}$ ahead of the node $\Omega_{7}$ and the reference frame has its equator inclined by $I_{7}$ with respect to the ecliptic plane. The initial rotation period $P$ is short such that $|\kappa|<\kappa_{\star}$ for the $s_{7}$ frequency. As a result the pole evolution is just a simple circulation about the Cassini state 2 (leftmost panel). As $P$ becomes longer, the critical $\kappa_{\star}$ value is reached and Cassini state $C_{2}$ arises at about $450 \mathrm{Myr}$ in our simulation. Because the associated resonant zone initially 

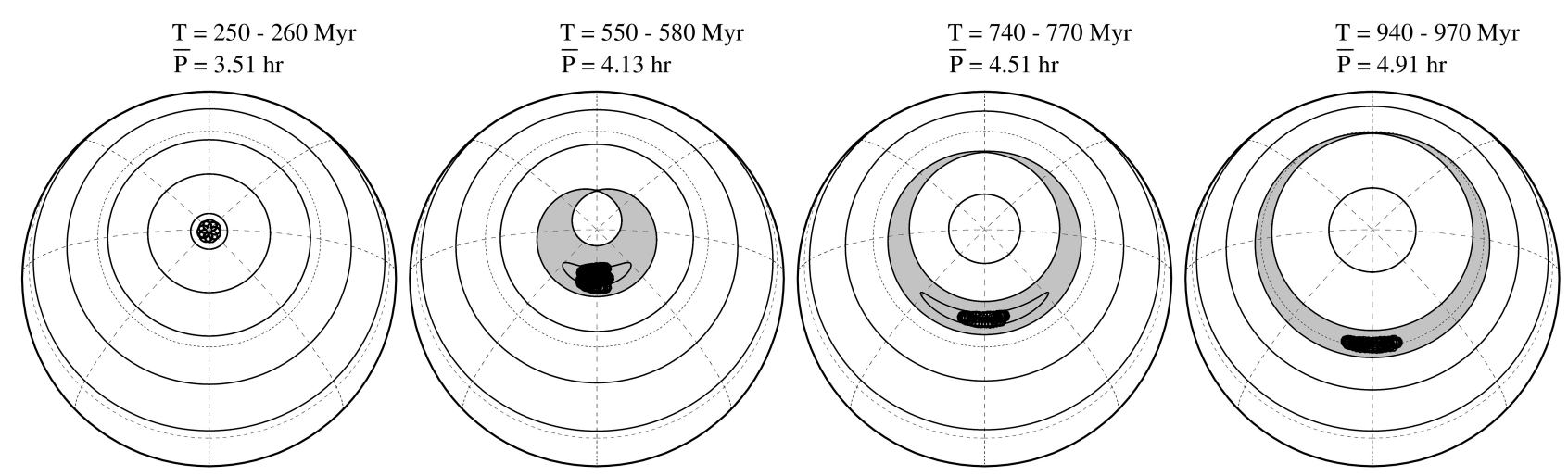

Fig. 5. Example of a successful capture of spin axis into the low-frequency Slivan state for a fictitious Themis member. Initially, the rotation axis orientation was identical to the north ecliptic pole (basically zero obliquity), and the rotation period $3 \mathrm{~h}$ was slowly increased by $2 \times 10^{-3} \mathrm{~h} / \mathrm{Myr}$. Spin axis evolution, represented as in Fig. 3, is shown for selected time intervals $(T)$ of our simulation noted at the top of each panel. The leftmost part shows the initial situation (no resonant zone about $\mathrm{C}_{2}$ for $s_{7}$ frequency), the rightmost part shows the final state (spin captured in a resonance zone about $\mathrm{C}_{2}$ at about $40^{\circ}$ obliquity, shown in gray). Rotation period at mean epoch of the displayed interval $(\bar{P})$ of time is given for each of the panels.

overlaps the ecliptic pole, the asteroid spin necessarily becomes captured in the resonance. The steady increase of $P$ makes $\mathrm{C}_{2}$, and its resonant zone, move to larger obliquity values. In this process the asteroid spin axis is safely dragged along.

Obviously, at a later stage of the evolution the resonance confinement should break because the resonance width $\Delta \varepsilon$ shrinks as $\varepsilon_{2}$ increases. Near adiabaticity of the evolution implies that the libration amplitude should increase (as also shown in our simulation), shifting the resonance librations closer to the separatrix. At a certain moment, the perturbations due to other than $s_{7}$ terms in $\zeta$ would destabilize the Slivan state. To study this process in detail, we would need to refine our model. For instance, the assumption that $\mathrm{d} P / \mathrm{d} t$ is constant is not exactly true because the spin period should typically stall somewhere between $\varepsilon_{2} \sim 40^{\circ}-50^{\circ}$ (e.g., Bottke et al. 2006). However, we note that the purpose of our experiment was only to verify the possibility of a quasi-stable capture in the low-frequency Slivan state rather than to present a detailed modeling of the complete spin evolution. We believe the results shown here clearly document this possibility.

\section{Conclusion}

Rotation of small asteroids in the main belt may occupy diverse states, some being more colorful than a simple regular precession due to the solar torque because their orbits precess in the inertial space due to planetary perturbations. The orbit precession periods are comparable to the estimated spin precession periods and this could result in resonant phenomena. Previously, researchers described such resonances with the leading $s_{6}$ mode of the orbit precession and called them Slivan states. Here we showed that the class of Slivan states may be broader since it could also contain situations when the spin precession is in resonance with the lower-frequency $s_{7}$ mode of the orbit precession. We find that the distant Themis family represents an ideal population of asteroids where these low-frequency Slivan states could be sought in the future. Alternatively, one could also consider the Schubart family among Hilda asteroids (e.g., Brož \& Vokrouhlický 2008), or even the low-inclination population among Jupiter Trojans. The latter is not surprising, because Jupiter's spin axis itself is possibly pushed to its $\simeq 3^{\circ}$ obliquity by the $s_{7}$ frequency (e.g., Ward \& Hamilton 2004; Vokrouhlický \& Nesvorný 2015). Spin states of asteroids residing on higher-inclination orbits in the outer belt among Hildas or Trojans are stirred by the orbit precession term associated with proper frequency $s$, and therefore they are not good candidates for the search of Slivan states.

Acknowledgements. This work was supported by the Czech Science Foundation (grant GA13-01308S).

\section{References}

Bertotti, B., Farinella, P., \& Vokrouhlický, D. 2003, Physics of the Solar System - Dynamics and Evolution, Space Physics, and Spacetime Structure. (Dordrecht: Kluwer Academic Press)

Bottke, W. F., Vokrouhlický, D., Rubincam, D. P., \& Nesvorný, D. 2006, Ann. Rev. Earth Planet. Sci., 34, 157

Boué, G., Laskar, J., \& Kuchynka, P. 2009, ApJ, 702, L19

Breiter, S., Nesvorný, D., \& Vokrouhlický, D. 2005, AJ, 130, 1267

Brož, M., \& Vokrouhlický, D. 2008, MNRAS, 390, 715

Bus, S. J., \& Binzel, R. P. 2002, Icarus, 158, 146

Colombo, G. 1966, AJ, 71, 891

Ďurech, J., Sidorin, V., \& Kaasalainen, M. 2010, A\&A, 513, A46

Durech, J., Carry, B., Delbò, M., Kaasalainen, M., \& Viikinkoski, M. 2015, in Asteroids IV, eds. P. Michel, F. E. DeMeo, \& W. F. Bottke, 183

Ferraz-Mello, S. 1981, AJ, 86, 619

Hamilton, D. P., \& Ward, W. R. 2004, AJ, 128, 2510

Henrard, J., \& Murigande, C. 1987, Celest. Mech., 40, 345

Kryszczyńska, A. 2013, A\&A, 551, A102

Laskar, J. 1988, A\&A, 198, 341

Marciniak, A., Michałowski, T., Kaasalainen, M., et al. 2007, A\&A, 473, 633

Masiero, J. R., Mainzer, A. K., Grav, T., et al. 2011, ApJ, 741, 68

Nesvorný, D., Brož, M., \& Carruba, V. 2015, in Asteroids IV, eds. P. Michel, F. E. DeMeo, \& W. F. Bottke, 297

Palisa, J. 1878, Astron. Nachr., 93, 197

Palisa, J. 1879, Astron. Nachr., 95, 291

Skoglöv, E. 1997, Planet. Space Sci., 45, 439

Skoglöv, E. 1998, Planet. Space Sci., 47, 11

Skoglöv, E., Magnusson, P., \& Dahlgren, M. 1996, Planet. Space Sci., 44, 1177

Slivan, S. M. 2002, Nature, 419, 49

Slivan, S. M., Binzel, R. P., Kaasalainen, M., et al. 2009, Icarus, 200, 514

Tedesco, E. F., Noah, P. V., Noah, M., \& Price, S. D. 2002, AJ, 123, 1056

Usui, F., Kuroda, D., Müller, T. G., et al. 2011, PASJ, 63, 1117

Vokrouhlický, D., \& Čapek, D. 2002, Icarus, 159, 449

Vokrouhlický, D., \& Nesvorný, D. 2015, ApJ, 806, 143

Vokrouhlický, D., Nesvorný, D., \& Bottke, W. F. 2003, Nature, 425, 147

Vokrouhlický, D., Bottke, W. F., \& Nesvorný, D. 2005, Icarus, 175, 419

Vokrouhlický, D., Nesvorný, D., \& Bottke, W. F. 2006, Icarus, 184, 1

Vraštil, J., \& Vokrouhlický, D. 2015, A\&A, 579, A14

Ward, W. R., \& Hamilton, D. P. 2004, AJ, 128, 2501

Ward, W. R., \& Canup, R. M. 2006, ApJ, 640, L91

Warner, B. D., Harris, A. W., \& Pravec, P. 2009, Icarus, 202, 134 